\title{
Size and albedo of the largest detected Oort-cloud object: Comet $\mathrm{C} / 2014 \mathrm{UN}_{271}$ (Bernardinelli-Bernstein)
}

\author{
E. Lellouch ${ }^{1} \odot$, R. Moreno ${ }^{1}$, D. Bockelée-Morvan ${ }^{1}$, N. Biver ${ }^{1}$, and P. Santos-Sanz ${ }^{2}$ \\ ${ }^{1}$ LESIA, Observatoire de Paris, PSL Research University, CNRS, Sorbonne Université, UPMC Univ. Paris 06, Univ. Paris Diderot, \\ Sorbonne Paris Cité, 5 place Jules Janssen, 92195 Meudon, France \\ e-mail: emmanuel.lellouch@obspm.fr \\ 2 Instituto de Astrofísica de Andalucía-CSIC, Glorieta de la Astronomía s/n, 18008 Granada, Spain
}

Received 12 January 2022 / Accepted 31 January 2022

\begin{abstract}
Context. The recently announced Oort-cloud comet C/2014 $\mathrm{UN}_{271}$ (Bernardinelli-Bernstein) is remarkable in at least three respects: (i) it was discovered inbound as far as $\sim 29$ au from the Sun (with prediscovery images up to $\sim 34$ au); (ii) it already showed cometary activity at almost $24 \mathrm{au}$; and (iii) its nuclear magnitude $\left(H_{r} \sim 8.0\right)$ indicates an exceptionally large object. Detection of gases is expected in the upcoming years as the comet heads toward a perihelion of $\sim 11$ au in 2031 .

Aims. The goal is to determine the object's diameter and albedo from thermal measurements.

Methods. We used ALMA in extended configuration (resolution 0.064") to measure the $1287 \mu \mathrm{m}(233 \mathrm{GHz})$ continuum flux of the comet. Observations were performed on August 8, 2021, at a 20.0 au distance from the Sun. The high spatial resolution was chosen in order to filter out any dust contribution. We also used a recently published $A f \rho$ value to estimate the dust production rate and the expected dust thermal signal for various assumptions on particle size distribution.

Results. We detected the thermal emission of the object at $\sim 10 \sigma$, with a flux of $0.128 \pm 0.012 \mathrm{mJy}$. Based on observational constraints and our theoretical estimates of the dust contribution, the entirety of the measured flux can be attributed to the nucleus. From NEATM modeling combined with the $H_{r}$ magnitude, we determine a surface-equivalent diameter of $137 \pm 17 \mathrm{~km}$ and a red geometric albedo of $5.3 \pm 1.2 \%$. This confirms that $\mathrm{C} / 2014 \mathrm{UN}_{271}$ is by far the largest Oort-cloud object ever found (almost twice as large as comet $\mathrm{C} / 1995$ O1 Hale-Bopp) and, except for the Centaur 95P/Chiron, which shows outburst-like activity, the largest known comet in the Solar System. On the other hand, the $\mathrm{C} / 2014 \mathrm{UN}_{271}$ albedo is typical of comets, adding credence to a "universal" comet nucleus albedo. Conclusions. With its distant perihelion and uniquely large size, $\mathrm{C} / 2014 \mathrm{UN}_{271}$ (Bernardinelli-Bernstein) is the prominent archetype of distant comets whose activity is driven by hypervolatiles. Monitoring of dust and gas emission as the comet approaches and passes perihelion will permit its activity time pattern to be studied and compared to the distant (outbound) activity of Hale-Bopp. Post-perihelion thermal measurements will permit the study of possible albedo changes, such as a surface brightening compared to pre-perihelion, as was observed for Hale-Bopp.
\end{abstract}

Key words. comets: individual: C/2014 UN271 (Bernardinelli-Bernstein)

\section{Introduction}

Comet C/2014 UN 271 (Bernardinelli-Bernstein) (hereafter 2014 $\mathrm{UN}_{271}$ for brevity) was discovered as part of the search for outer Solar System objects with the Dark Energy Survey (DES) performed over the period 2013-2019 (Bernardinelli et al. 2021a). $2014 \mathrm{UN}_{271}$ was observed in 42 DES survey images on 25 nights from October 2014 to November 2018, with a heliocentric distance $r_{h} \sim 29$ au (Bernardinelli et al. 2021b). Prediscovery images from WISE, CFHT, VST, VISTA, and Pan-STARRS extend the photometric record to October $2010\left(r_{h} \sim 34.1 \mathrm{au}\right)$. Orbital analysis (Bernardinelli et al. 2021b) indicates characteristic Oort-cloud membership, with an inclination and a semimajor axis of the incoming orbit of $95.5^{\circ}$ and $20200 \mathrm{au}$, respectively (i.e., an inbound orbital period of $\sim 2.9$ million years). The object is heading toward a 10.95 au perihelion passage, to occur on January 21, 2031. Backward orbit integration points to a previous perihelic passage at 17-21 au and suggests that $2014 \mathrm{UN}_{271}$ has never been closer than this distance since its ejection from the
Oort cloud, possibly making it one of the most "pristine" comets ever observed.

The announcement of the object on June 19, 2021 (Bernardinelli \& Bernstein 2021) prompted immediate observations, which showed a visible coma at $r_{h}=20.18$ au (Demetz et al. 2021; Kokotanekova et al. 2021; Buzzi \& Lister 2021). Analysis of Transiting Exoplanet Survey Satellite (TESS) data from September and October 2018 indicated that a coma was already present at $23.8 \mathrm{au}$, and syndyne analysis suggested the activity might have started several years earlier (Farnham et al. 2021). $2014 \mathrm{UN}_{271}$ thus joins the list of inbound, distantly active, long-period comets that includes C/2017 K2 (Pan-STARRS), C/2010 U3 (Boattini), and C/2014 B1 (Schwartz). Also, comet C/1995 O1 Hale-Bopp was observed to be active and outbound 11 years after perihelion at 25.7 au from the Sun (Szabó et al. 2008) and may be even have been at 30.7 au (Szabó et al. 2011). Activity in these objects may in fact occur even farther away: modeling of the $r_{h}$ dependence of the dust production rate in $\mathrm{C} / 2017 \mathrm{~K} 2$ indicates activity was already 
Table 1. Observation parameters.

\begin{tabular}{|c|c|c|c|c|c|c|c|c|}
\hline \multirow{3}{*}{$\begin{array}{l}\text { Scheduling } \\
\text { block } \\
\text { SB1 }\end{array}$} & \multirow{3}{*}{$\begin{array}{c}\begin{array}{c}\text { UT date } \\
\text { (start/end) }\end{array} \\
\begin{array}{c}\text { 08-Aug-2021 } \\
7: 37-8: 35\end{array}\end{array}$} & \multirow{3}{*}{$\begin{array}{c}\begin{array}{c}\text { Integration } \\
\text { time }\end{array} \\
1624 \mathrm{sec}\end{array}$} & \multirow{2}{*}{\multicolumn{2}{|c|}{$\begin{array}{l}\text { Flux calibrator } \\
\text { and flux density }{ }^{(a)}\end{array}$}} & \multirow{2}{*}{\multicolumn{2}{|c|}{$\begin{array}{l}\text { Phase calibrator } \\
\text { and flux density } \\
\left({ }^{b}\right)\end{array}$}} & \multicolumn{2}{|c|}{$2014 \mathrm{UN}_{271}$} \\
\hline & & & & & & & \multirow{2}{*}{$\begin{array}{c}\text { Flux density }^{(b)} \\
0.109 \pm 0.016 \mathrm{mJy}\end{array}$} & $(\Delta \mathrm{RA}, \Delta \mathrm{Dec})^{(c)}$ \\
\hline & & & $\mathrm{J} 2258-2758$ & $1.22 \mathrm{Jy}$ & J0253-5411 & $0.374 \mathrm{Jy}$ & & $-0.091^{\prime \prime}-0.249^{\prime \prime}$ \\
\hline SB2 & $\begin{array}{c}\text { 08-Aug-2021 } \\
8: 35-9: 30\end{array}$ & $1613 \mathrm{sec}$ & J0519-4546 & $1.26 \mathrm{Jy}$ & J0253-5411 & $0.389 \mathrm{Jy}$ & $0.147 \pm 0.016 \mathrm{mJy}$ & $-0.100^{\prime \prime}-0.262^{\prime \prime}$ \\
\hline
\end{tabular}

Notes. ${ }^{(a)}$ Assumed. ${ }^{(b)}$ Measured. ${ }^{(c)}$ (RA, Dec) offset with respect to predicted position. Adopting a mean $\left(-0.0955^{\prime \prime},-0.2555^{\prime \prime}\right)$ offset for the two SBs, the J2000 astrometric coordinates with respect to the observing site (Code -7 ) on August 8, 2021, UT = 8:00 are: RA =02:42:04:2508 and $\mathrm{Dec}=-53: 26: 48.520$.

present at $35 \mathrm{au}$, presumably driven by the sublimation of $\mathrm{CO}$ or other supervolatile ices (Jewitt 2021).

Of further interest for the characterization of $2014 \mathrm{UN}_{271}$ are the following aspects (Ridden-Harper et al. 2021; Farnham et al. 2021; Bernardinelli et al. 2021b; Kokotanekova et al. 2021): (i) possible fluctuations in the magnitude but a lack of a clear rotational signal at the $>0.2-0.3 \mathrm{mag}$ level; and (ii) a moderately red color, with spectral slope $5-10 \% / 100 \mathrm{~nm}$, typical of (or slightly bluer than) long-period comets (Jewitt 2015). Gas emissions have not yet been detected, with production rates $Q_{\mathrm{CO}}<1.25 \times 10^{28} \mathrm{~mol} \mathrm{~s}^{-1}$ and $Q_{\mathrm{CN}}<1.25 \times 10^{27} \mathrm{~mol} \mathrm{~s}^{-1}$ near $\sim 21$ and $\sim 20$ au, respectively (Farnham et al. 2021; Kokotanekova et al. 2021). Most remarkable for $2014 \mathrm{UN}_{271}$, however, is its exceptionally bright absolute magnitude, $H_{r}=7.96 \pm 0.03$, which showed no evolution in ground-based data during the approach from 34 to 22 au and therefore presumably represents the nuclear magnitude (Bernardinelli et al. 2021b). For a standard cometary 5\% red albedo, and based on usual relationships between diameter $(D)$ and $H$ magnitude (see Sect. 3.2), this yields $D=130 \mathrm{~km}$, potentially making $2014 \mathrm{UN}_{271} 1.75$ times larger than Hale-Bopp $(74 \pm 6 \mathrm{~km}$ diameter; Szabó et al. 2012). $2014 \mathrm{UN}_{271}$ would thus compete in size with the largest Centaurs and with the smallest of the trans-Neptunian objects (TNOs) for which size measurements are available; however, the typical (visible) albedos of TNOs are typically around 8\% (Müller et al. 2020). Based on Hale-Bopp experience (Biver et al. 2002; Rauer et al. 2003), detection of $\mathrm{CO}, \mathrm{CN}$, and other species in $2014 \mathrm{UN}_{271}$ may be expected as it heads toward and beyond its $\sim 11$ au perihelion in the upcoming years, and a determination of its diameter and albedo is needed to complete its physical characterization. We report here on such measurements based on the detection of thermal emission with the Atacama Large Millimeter Array (ALMA) and the application of the radiometric technique.

\section{Observations and data reduction}

Observations of comet $\mathrm{C} / 2014 \mathrm{UN}_{271}$ were obtained on August 8, 2021, with the 12-meter array of ALMA, under the ALMA DDT (Director Discretionary Time) project 2019.A.00038 (see details in Table 1). Heliocentric distance, geocentric distance, and phase angle values of the target were $r_{h}=20.0046 \mathrm{au}$, $\Delta=19.6776 \mathrm{au}$, and $\alpha=2.77^{\circ}$, respectively. All observations were taken in ALMA Band $6(211-275 \mathrm{GHz})$ in the continuum ("TDM") mode. We used the standard frequency tuning for that band, which yielded four $1.875-\mathrm{GHz}$ broad spectral windows centered at 224, 226, 240, and $242 \mathrm{GHz}$. The array was in extended configuration $\mathrm{C} 8$, with 40 operating antennas. This yielded baselines in the range $92 \mathrm{~m}-8282 \mathrm{~m}$ and a synthesized beam of $\sim 0.065^{\prime \prime}$ for robust weighting $(0.5)$, which is larger than the expected nucleus size ( $\sim 10$ mas) but allows dust emission to be filtered out. Observations were obtained in dual polarization mode, with the two polarizations combined at the data reduction stage to provide a measurement of the total flux.

The observations consisted of two scheduling blocks (SBs), each $\sim 55-58 \mathrm{~min}$ long, including $\sim 27 \mathrm{~min}$ on source. The rest of the time in each SB was spent on flux (bandpass) calibrators and on secondary (phase) calibrators for monitoring the atmospheric and instrumental amplitude and phase gains. Observations occurred in excellent weather conditions (zenithal precipitable water vapor $\sim 0.9 \mathrm{~mm}$; antenna-based phase rms $\sim 26$ degrees). Observational details and flux results are given in Table 1.

The flux calibrators, namely quasars J0519-4546 and J22582758 for the two SBs, are variable but routinely monitored. Details on how we estimated their flux and associated error bar on the observing date are given in Appendix A.

Initial steps of the data reduction were performed in the CASA reduction package via the ALMA pipeline (Muders et al. 2014), which provided a set of visibilities as a function of baseline between each antenna pair. Visibility fitting was performed for the two flux calibrators, and visibilities were rescaled (by factors of 0.99 and 0.995 for SB1 and SB2, respectively, from the ALMA pipeline calibration) so that their measured flux matched the values expected from Appendix A (and recalled in Table 1). These factors were also applied to visibility data of the phase calibrator (J0253-5441) and of the science target. Visibility fitting provided two independent values of the flux of J0253-5441 that were found to be consistent within $4 \%$ (see Table 1), confirming the quality of the flux scale.

For $2014 \mathrm{UN}_{271}$, visibilities were exported into the GILDAS package for imaging and visibility fitting. Combining data from the two SBs and the four spectral windows resulted in the $233 \mathrm{GHz}$ image of $\mathrm{C} / 2014 \mathrm{UN}_{271}$ shown in Fig. 1 and yielded a detection of the object at $\sim 10 \sigma$. Visibility fitting was performed independently for the two SBs. In a first step, for each $\mathrm{SB}$, each of the four spectral windows yielded a measure of the flux and of the (RA, Dec) position offset of the target from the expected ephemeris ${ }^{1}$, leaving these parameters free in the fit and specifying a point-like source. The four sets of (RA, Dec) values were then kept fixed at their average values (see Table 1), and the visibilities were refit in terms of the object total flux. We specified either a point-like source or a 9.5 milliarcsecond (mas) disk (which corresponds to the a posteriori determination of the object diameter), with insignificant differences $(\sim 0.001 \mathrm{mJy}$ in flux). For each SB, the combined $233 \mathrm{GHz}$ flux and its error bar were obtained by merging the visibilities from the four spectral windows (GILDAS task uv_merge) after rescaling to that frequency using a spectral index $\alpha=\mathrm{d}\left(\log F_{v}\right) / \mathrm{d}(\log$ $v$ ) of 1.93 in the $224-242 \mathrm{GHz}$ range, as expected from NEATM

1 JPL/Horizons interrogated on July 14, 2021. 


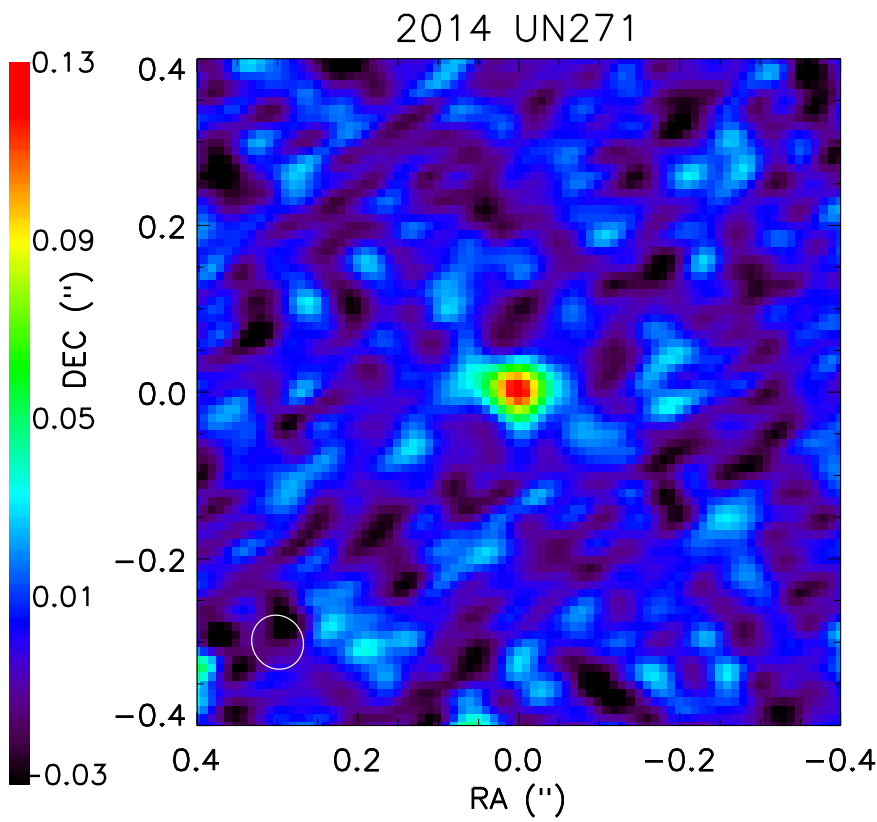

Fig. 1. Re-centered $233 \mathrm{GHz}$ total image of $2014 \mathrm{UN}_{271}$, merging data from the two SBs and the four spectral windows. The synthesized beam, shown in white, is $0.067^{\prime \prime} \times 0.062^{\prime \prime}$. The scale of the color bar is in mJy.

models (see Sect. 3.2), and performing visibility fitting again. This yielded $0.109 \pm 0.016 \mathrm{mJy}$ for SB1 and $0.147 \pm 0.016 \mathrm{mJy}$ for SB2, which is a somewhat unexpected $2.4 \sigma$ difference. In theory, the object's thermal flux might show rotational variability associated with a triaxial $(a, b, c)$ projected shape, but even assuming the two SBs were precisely in phase with projected surface maxima and minima, the $35 \%$ higher flux in SB2 would strictly imply $a>1.8 b$. This is also at face value inconsistent with the lack of rotational variability at the $>0.2-0.3$ magnitude level in optical data, although optical variability might be damped by coma contribution. In what follows, we simply averaged the fluxes from the two SBs, which yielded $0.128 \pm 0.011 \mathrm{mJy}^{2}$. Quadratically adding a conservative $4 \%$ uncertainty on the flux calibrator scale, the final object flux is $0.128 \pm 0.012 \mathrm{mJy}$.

\section{Analysis}

\subsection{Estimate of coma contribution}

Visibility curves, $V$, as a function of UV radius, $\sigma$, bear information on the spatial distribution of the source. For a uniform disk of apparent diameter $\theta, V(\sigma)$ follows a $\mathrm{J}_{1}$ Bessel function, with first zero at $\sigma_{0}=1.22 \lambda / \theta$ and a constant value for a point-like source. Extended emission for a coma brightness distribution varying as $1 / \rho$, where $\rho$ is the distance to comet center, shows up as visibilities $V(\sigma) \propto 1 / \sigma$ (Bockelée-Morvan et al. 2010). Figure 2 shows the real part of the observed visibilities for $2014 \mathrm{UN}_{271}$, weight-averaged in 400-meter-wide $\sigma$ bins plotted in terms of $\lambda$. From $\chi^{2}$ analysis, visibility fitting cannot distinguish between disk sizes smaller than $\sim 40$ mas, which is to be expected given the $\sim 65$ mas resolution achieved and the measurements' $\mathrm{S} / \mathrm{N}$. Most importantly, within the noise level, no indication of a $1 / \sigma$ signal component is apparent. Fitting the

\footnotetext{
2 This error bar is also satisfactorily consistent with $\operatorname{stdev} / \sqrt{8}=$ $0.013 \mathrm{mJy}$, where stdev is the standard deviation between the eight individual flux values rescaled to $233 \mathrm{GHz}$
}

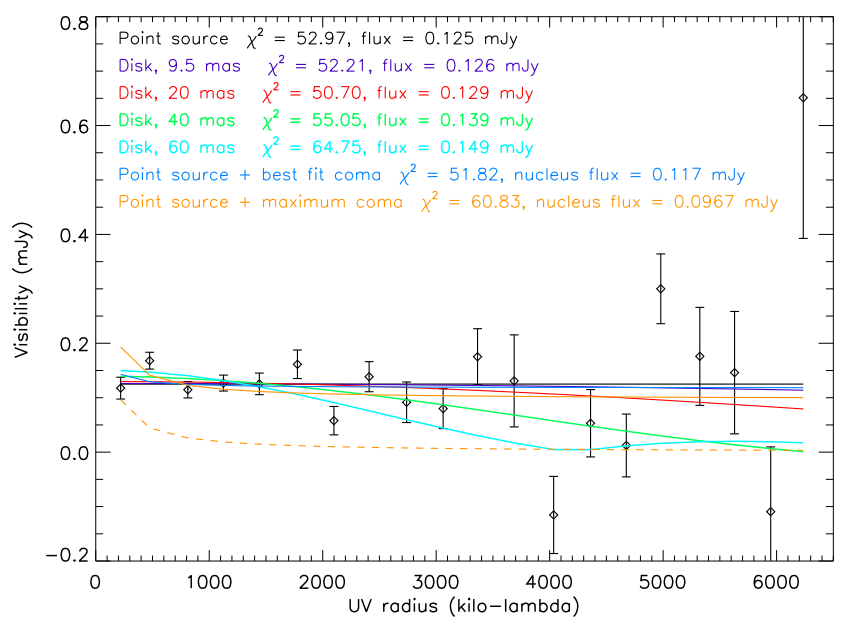

Fig. 2. Real part of the visibilities, averaged in 400-meter UV radius bins, compared to various models: (i) point source, (ii) disks with apparent sizes of 9.5, 20, 40, and 60 mas, and (iii) sum of a point source and a coma. The visibilities are rescaled to $233 \mathrm{GHz}$, and the UV radii are expressed in terms of the wavelength (kilo- $\lambda$ ). The dashed orange curve indicates the strongest coma signal that can be accommodated by the data.

$V(\sigma)$ curve with the sum of a constant and a $1 / \sigma$ term provides no better fit than the previous point-like and $<40$ mas disk models, and in this case (sixth model in Fig. 2, blue curve), the nucleus contributes $93 \%$ of the signal. A $3 \sigma$ upper limit to the contribution of the coma to the visibility at $\sigma=270 \mathrm{~m}(220 \mathrm{k} \lambda)$ can be set at the $0.1 \mathrm{mJy}$ level (orange curves in Fig. 2). Even in this extreme case, the contribution of the nucleus is $0.097 \mathrm{mJy}$ (i.e., $76 \%$ of the total flux). This is a first, observational piece of evidence of a dominant nuclear contribution to the signal.

Furthermore, based on the comet dust activity level reported on June 29, 2021 (Dekelver 2021; Bernardinelli et al. 2021b), an $A f \rho$ value of $\sim 150 \mathrm{~m}$, we estimated the expected thermal signal from the dust in the ALMA synthesized beam for different assumptions of the particle size index $(\beta)$ and maximum particle radius $\left(a_{\max }\right)$. Results, described in Appendix B, indicate that for most assumptions the thermal emission of dust is entirely insignificant. Only if the particle size distribution is extremely shallow $(\beta=3)$ and the maximum particle size very large $\left(a_{\max }=1 \mathrm{~cm}\right)$ is there a non-negligible, albeit still minor and within the uncertainty of the measured visibilities, dust contribution to the measured signal. In the rest of the paper, the measured thermal flux of $0.128 \pm 0.012 \mathrm{mJy}$ is attributed to the nucleus only.

\subsection{Nucleus diameter and albedo}

Without knowledge of nucleus shape and spin parameters (pole orientation and shape), a thermophysical model is pointless, and we instead adopted a NEATM (Near Earth Asteroid Thermal Model) model, used extensively for asteroids (Harris 1998) and TNOs (Müller et al. 2020, and references therein). NEATM is based on the asteroid standard thermal model (STM; Lebofsky et al. 1989) but accounts for phase angle effects; additionally, the temperature distribution is modified by an adjustable $\eta^{-1 / 4}$ factor, which represents the combined and opposed effects of roughness $(\eta<1)$ and thermal inertia $(\eta>1)$. For fixed surface (thermal inertia, roughness) and spin properties, $\eta$ is also a function of the subsolar temperature, and, therefore, of the heliocentric distance (e.g., Spencer et al. 1989; 
Lellouch et al. 2013). Given the $r_{h}=20$ au distance of our measurements (and the expected large size of $2014 \mathrm{UN}_{271}$ ), we adopted a beaming factor $\eta=1.175 \pm 0.42$, based on measurements of 85 Centaurs and TNOs (Lellouch et al. 2013, 2017). We also specified a bolometric emissivity $\epsilon_{b}=0.90 \pm 0.06$ and a relative radio emissivity $\epsilon_{r}=\epsilon_{m m} / \epsilon_{b}=0.70 \pm 0.13$, as inferred from combined Spitzer/Herschel/ALMA measurements of nine objects (Brown \& Butler 2017; Lellouch et al. 2017). The lowerthan-unity relative radio emissivity is interpreted as resulting from (i) the sounding of a colder dayside subsurface and (ii) the loss of outgoing thermal radiation due to volume scattering in the subsurface and/or Fresnel reflection at the surface. The few available radio observations of cometary nuclei also generally indicate radio emissivities lower than 1 , for example $\sim 0.5$ for Hale-Bopp (Fernández 2002) and $<0.8$ for $8 \mathrm{P} /$ Tuttle (Boissier et al. 2011). Comets are also found to have low thermal inertias (e.g., $<10,<30$, and $<45 \mathrm{MKS}$ for $8 \mathrm{P} /$ Tuttle, 22P/Kopff, and 9P/Tempel 1, respectively; Boissier et al. 2011; Groussin et al. 2009, 2013), consistent with a beaming factor, $\eta$, of order unity. Based on NEATM analysis of a large sample of comet nuclei observed with Spitzer at $r_{h}=3.5-$ 6 au, Fernández et al. (2013) find a mean $\eta$ of $1.03 \pm 0.11$. The large $29 \mathrm{P} /$ Schwassmann-Wachmann nucleus $(D=65 \mathrm{~km})$ has $\eta=1.1 \pm 0.2$ (Schambeau et al. 2021). These numbers are fully consistent with our choice of $\eta$. Given the values of $r_{h}, \eta$, and $\epsilon_{b}$, NEATM calculations indicate that the object's spectral index over $224-242 \mathrm{GHz}$ is 1.93 , slightly lower than the RayleighJeans limit of 2.

With the above parameters, the measured thermal flux yields the object's (surface-equivalent) diameter, $D$, and the albedo was then determined from the usual relationship between diameter $(D)$ and magnitude $(H), D=2 a / \sqrt{p} 10^{0.2\left(m_{\odot}-H\right)}$, where $p$ is the object's geometric albedo, $m_{\odot}$ is the solar magnitude in the relevant band, and $a=1 \mathrm{au}$. Using $V_{\odot}=-26.76$ and $(V-$ $R)_{\odot}=0.35$, one obtains $D=1330 \mathrm{~km} / \sqrt{p_{V}} 10^{-0.2 H_{V}}$ from the $V$ band and $D=1132 \mathrm{~km} / \sqrt{p_{R}} 10^{-0.2 H_{R}}$ from the $R$ band. We converted the griz magnitudes and colors from Bernardinelli et al (2021b) using the prescriptions of Jester et al. (2005), which yields $H_{V}=8.21 \pm 0.05, H_{R}=7.75 \pm 0.05$, and $V-R=0.46 \pm$ 0.02 (i.e., $p_{R}=1.11 \pm 0.02 p_{V}$ ). The temperature distribution, $T$, across the object is also a function of the object's albedo, $p_{V}$, through $T \propto\left(1-p_{V} q\right)^{1 / 4}$ (where a reasonable value of $q$, the phase integral, is $\sim 0.4$ ). However, this dependence is minimal given the a posteriori low albedo inferred $\left(p_{V} \sim 0.049\right)$, so iterating once on the albedo in NEATM was sufficient.

To account for uncertainties, both on the measured object's flux and on the model inputs $\left(\eta, \epsilon_{b}, \epsilon_{r}\right.$, and $\left.H_{r}\right)$, we randomly generated a large set (40000) of synthetic data and model inputs, based on Gaussian-added noise on each parameter at the appropriate level (Mueller et al. 2011), solving for $D$ and $p_{R}$ in each case. In doing so, we restricted $\eta$ values to be $>0.6$ (lower values are physically implausible; see Mommert et al. 2012), as well as $\epsilon_{b}<1$ and $\epsilon_{r}<1$.

\section{Results and discussion}

Figure 3 shows the distribution of the solution $D$ and $p_{R}$, accounting separately for flux uncertainties, model uncertainties, and both. This provided best-fit values and error bars (defined to include the central $68.3 \%$ of the results) for the diameter and albedo. We find $D=137 \pm 17 \mathrm{~km}^{3}$ and

\footnotetext{
3 This yields an apparent diameter of 9.6 mas at $\Delta=19.68 \mathrm{au}$, consistent with the point-like appearance of the object in the visibility curve (Fig. 2).
}
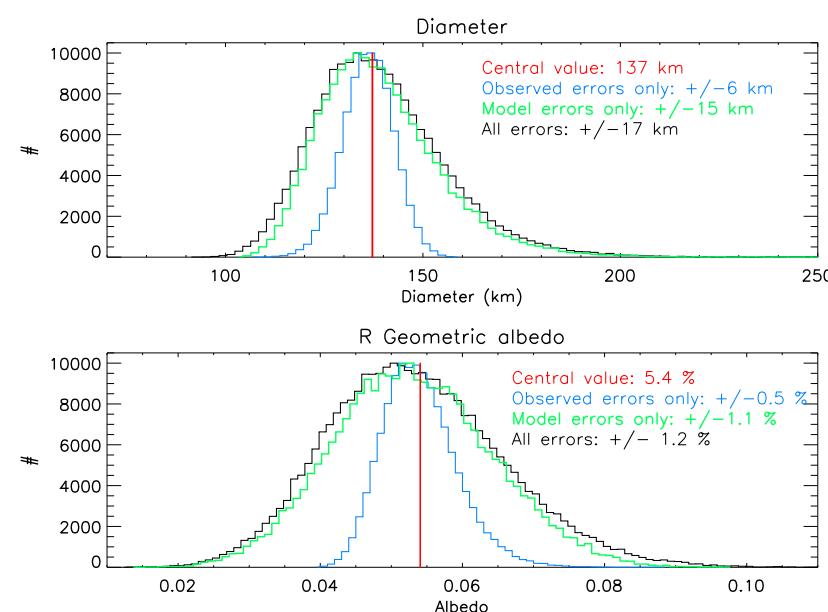

Fig. 3. Diameter and $\mathrm{R}$ albedo distribution of solutions. The red line shows the central values. Blue, green, and black curves show the distributions associated with flux uncertainties, model uncertainties, and both, respectively. For an easier comparison, all distributions are rescaled to peak at 10000 .

$p_{R}=5.3 \pm 1.2 \%$, where error bars are dominated by model errors - and principally by the uncertainty on $\epsilon_{r}$. This confirms that $2014 \mathrm{UN}_{271}$ is almost twice larger in diameter than Hale-Bopp and makes it the largest Oort-cloud comet detected so far. $2014 \mathrm{UN}_{271}$ is also larger than almost all active Centaurs (e.g., $D \sim 60 \mathrm{~km}$ for $167 \mathrm{P} /$ Cineos, 174P/Echeclus, and 29P/Schwassmann-Wachmann; see Müller et al. 2020; Schambeau et al. 2021), being surpassed only by $95 \mathrm{P} / \mathrm{Chiron}$ ( $D \sim 215 \mathrm{~km}$; Fornasier et al. 2013). Given, however, that Centaurs activity occurs mostly in the form of outbursts of variable lifetime ${ }^{4}$ but is mostly uncorrelated with heliocentric distance (Peixinho et al. 2020, and references therein), $2014 \mathrm{UN}_{271}$ appears as the largest "standard comet" ever discovered".

Unlike the size, the albedo of $2014 \mathrm{UN}_{271}, p_{V}=4.9 \pm 1.1 \%$, is completely in line with that of other, typically much smaller, comets $(2-6 \%$ in $V$ or $R$ for a sample of $\sim 80$ ecliptic or near-isotropic comets, with no discernible trends with other orbital or physical parameters; see Lamy et al. 2004; Campins \& Fernández 2002). Our measurement thus adds evidence against a dependence of comet nucleus albedo on size. Such a conclusion was reached previously by Fernández et al. (2013) on the basis that the size distribution they measured for 89 comets in the thermal range is indistinguishable from that inferred from optical photometry assuming constant albedo. In this context, 95P/Chiron, with $p_{V}=0.10-0.17$ (Lellouch et al. 2017), stands as a clear outlier both from comet nuclei and from the Centaur population as a whole (median $p_{V}, 5.6 \%$; see Müller et al. 2020) ${ }^{6}$. We also note that with its $\sim 5 \%$ albedo and 5-10\%/100 nm spectral slope, $2014 \mathrm{UN}_{271}$ falls in the middle of the "dark/neutral" cluster identified in the Kuiper Belt (Lacerda et al. 2014). The low albedos encountered on many outer Solar System objects are usually associated with the presence of exposed organics along with additional darkening agents, such as sulfides (Rousseau et al. 2018). This hypothesis

\footnotetext{
4 In the case of 29P, superimposed on a steady background activity level.

5 For 2014 UN $_{271}$, Kelley et al. (2021) reported activity variation possibly associated with outbursts, but the long-term behavior is more characteristic of continuous activity (Farnham et al. 2021).

6 We note that the geometric albedo of $29 \mathrm{P}$ is very uncertain, $p_{V}=2.5$ $12 \%$, depending on the adopted $H_{v}$ magnitude (Stansberry et al. 2004).
} 
is strengthened by the detection of large amounts $(\sim 50 \%$ in mass) of organics in the dust of 67P/Churyumov-Gerasimenko (Bardyn et al. 2017), but many questions remain as to the relations between albedo, color, composition, irradiation, and activity (see, e.g., Brunetto et al. 2006; Jewitt 2015; Poston et al. 2018; Wong et al. 2019).

Our observation at 20 au provides the most distant determination of the albedo of a new Oort-cloud object on its inbound orbit. This is of interest because cometary activity may cause nucleus albedo (and color) to change over time. In the case of comet Hale-Bopp, a joint analysis of pre- and postperihelion data indicated $p_{R} \sim(3.1-3.6) \pm 1.0 \%$ at 6.4 and 4.4 au inbound (Szabó et al. 2012), in agreement within errors with our determination for $2014 \mathrm{UN}_{271}$, but an exceptionally high $p_{R}=8.1 \pm 0.9 \%$ at $31-32$ au outbound. This was interpreted as due to gravitational redeposition of bright icy grains near the cessation of the outbound activity, burying low-albedo material. Although the mechanism was at the time deemed more likely to occur in large objects (favoring gravitational fallback) and with distant activity (associated with slower velocities), a similar redeposition mechanism was responsible for the bright, smooth, ejecta-covered "neck" (Hapi) region of comet 67P/ChuryumovGerasimenko ${ }^{7}$. Remeasuring the thermal emission (and colors) of $2014 \mathrm{UN}_{271}$ post-perihelion (e.g., at 20 au outbound in 2040) will permit the assessment of whether these processes occur as well on this comet.

Just as Hale-Bopp is the archetype of a large comet on a Sun-approaching orbit, $2014 \mathrm{UN}_{271}$ appears as the most prominent representative of distant, long-period comets whose activity is governed by hypervolatiles $\left(\mathrm{CO}, \mathrm{CO}_{2}\right.$, etc.), and monitoring of its chemical composition as it approaches and passes perihelion will be of high value. Scaling Hale-Bopp (outbound) activity data (Biver et al. 2002) by $D^{2}$ and $r_{h}^{-2}$, we expect a current CO production rate in $2014 \mathrm{UN}_{271}$ of $Q_{\mathrm{CO}}=7 \times 10^{27} \mathrm{~mol} \mathrm{~s}^{-1}$, rising to $\sim 2 \times 10^{28} \mathrm{~mol} \mathrm{~s}^{-1}$ at the $\sim 11$ au perihelion in January 2031 . Likewise, based on Hale-Bopp CN data up to 9.8 au (Rauer et al. 2003), we anticipate $Q_{\mathrm{CN}} \sim 2 \times 10^{25} \mathrm{~mol} \mathrm{~s}^{-1}$ at perihelion. While signals will remain modest, requiring the use of sensitive facilities (ALMA, VLT, JWST, etc.), both species and possibly a few others ( $\mathrm{HCN}, \mathrm{CH}_{3} \mathrm{OH}, \mathrm{CO}_{2}$, etc.), should be detectable and monitored over a $\sim 10$ year period around perihelion. The biggest difference between $2014 \mathrm{UN}_{271}$ and Hale-Bopp, however, is that the former will not enter the water-dominated activity regime; comparisons between the intrinsic (i.e., per $\mathrm{km}^{2}$ ) activity pattern (outbound, for Hale-Bopp) in the two comets, and possibly in some active Centaurs, will provide further insights into the mechanisms of distant cometary activity. Additional information on the spin properties, shape, and thermal regime of $2014 \mathrm{UN}_{271}$ should also be gained in the near future from combined optical imaging, JWST thermal measurements, and possibly stellar occultations.

Acknowledgements. This paper is based on ALMA program 2019.A.00038. ALMA is a partnership of ESO (representing its member states), NSF (USA) and NINS (Japan), together with NRC (Canada), NSC and ASIAA (Taiwan), and KASI (Republic of Korea), in cooperation with the Republic of Chile. The Joint ALMA Observatory is operated by ESO, AUI/NRAO and NAOJ. The National Radio Astronomy Observatory is a facility of the National Science Foundation operated under cooperative agreement by Associated Universities, Inc. P.S-S.

The nucleus of 67P also underwent blueing and brightening during the perihelion passage due to the blowing-off of volatile-depleted superficial layers and the exposure of brighter and bluer ice-rich subsurface layers (Fornasier et al. 2016; Filacchione et al. 2020). acknowledges financial support from the Spanish grant AYA-RTI2018-098657J-I00 "LEO-SBNAF" (MCIU/AEI/FEDER, UE) and from the State Agency for Research of the Spanish MCIU through the "Center of Excellence Severo Ochoa" award to the Instituto de Astrofísica de Andalucía (SEV-2017-0709).

\section{References}

A'Hearn, M. F., Schleicher, D. G., Millis, R. L., et al. 1984, AJ, 89, 579 Bardyn, A., Baklouti, D., Cottin, H., et al. 2017, MNRAS, 469, S712 Bernardinelli, P. H., \& Bernstein, G. 2021, Minor Planet Electronic Circulars, 2021-M53

Bernardinelli, P. H., Bernstein, G. M., Sako, M., et al. 2021a, AAS J., submitted [arXiv:2109.03758]

Bernardinelli, P. H., Bernstein, G. M., Montet, B., et al. 2021b, ApJ, 921, L37

Biver, N., Bockelée-Morvan, D., Colom, P., et al. 2002, Earth Moon Planets, 90, 323

Bockelée-Morvan, D., Boissier, J., Biver, N., \& Crovisier, J. 2010, Icarus, 210, 898

Bockelée-Morvan, D., Rinaldi, G., Erard, S., et al. 2017, MNRAS, 469, S443

Boissier, J., Groussin, O., Jorda, L., et al. 2011, A\&A, 528, A54

Brown, M. E., \& Butler, B. J. 2017, AJ, 159, 19

Brunetto, R., Barucci, M. A., Dotto, E., \& Strazzulla, G. 2006, ApJ, 644, 646

Buzzi, L., \& Lister, T. 2021, Minor Planet Electronic Circulars, 2021-M83

Campins, U., \& Fernández, Y. 2002, Earth Moon Planets, 89, 117

Crifo, J. F., \& Rodionov, A. V. 1997, Icarus, 127, 319

Dekelver, P.-J. 2021, C/2014 UN271 Bernardinelli-Bernstein. http: //wwW . astronomie.be/pieterjan.dekelver/Observations/ 20210629_C2014UN271_Bernardinelli-Bernstein_W86_FULL.jpg

Demetz, L., Buzzi, L., Aletti, A., \& Lister, T. 2021, Central Bureau Astronomical Telegrams, CBET-4989

Fink, U., \& Rubin, M. 2012, Icarus, 221, 721

Farnham, T. L., Kelley, M. S. P., \& Bauer, J. M. 2021, PSJ, 2, 236

Fernández, Y. R. 2002, Earth Moon Planets, 89, 3

Fernández, Y. R., Kelley, M. S., Lamy, P., et al. 2013, Icarus, 226, 1138

Filacchione, G., Capaccioni, F., Ciarniello, M., et al. 2020, Nature, 578, 49

Fornasier, S., Lellouch, E., Müller, T., et al. 2013, A\&A, 555, A15

Fornasier, S., Mottola, S., Keller, H.-U., et al. 2016, Science, 354, 1566

Groussin, O., Lamy, P., Toth, I., et al. 2009, Icarus, 199, 568

Groussin, O., Sunshine, J. M., Feaga, L. M., et al. 2013, Icarus, 222, 580

Harris, A. W. 1998, Icarus, 131, 291

Jester, S., Schneider, D. P., Richards, G. T., et al. 2005, AJ, 130, 873

Jewitt, D. 2015, AJ, 150, 201

Jewitt, D. 2021, AJ, 161, 188

Kelley, M. S. P., Lister, T., \& Holt, C. 2021, ATel, 14917, 1

Kokotanekova, R., Opitom, C., Knight, M., et al. 2021, Bull. Amer. Astron. Soc., 53, No. 7 e-id 2021n7i301p04

Lacerda, P., Fornasier, S., Lellouch, E., et al. 2014, ApJ, 793, 6

Lamy, P. L., Toth, I., Fernandez, Y. R., \& Weaver, H. A. 2004, in Comets II, eds. M. C. Festou, H. U. Keller, \& H. A. Weaver, 223

Lebofsky, L. A., \& Spencer, J. R. 1989, in Asteroids II, eds. R. P. Binzel,

T. Gehrels, \& M. S. Matthews (Arizona University Press), 128

Lellouch, E., Santos-Sanz, P., Lacerda, P., et al. 2013, A\&A, 557, A60

Lellouch, E., Moreno, R., Müller, T., et al. 2017, A\&A, 608, A45

Mommert, M., Harris, A. W., Kiss, Cs, et al. 2012, A\&A, 541, A93

Muders, D., Wyrowski, F., Lightfoot, J., et al. 2014, in The ALMA Pipeline, eds.

N. Manset, \& P. Forshay, Astronomical Data Analysis Software and Systems XXIII May 2014

Mueller, M., Delbo, M., Hora, J. L., et al. 2011, AJ, 141, 109

Müller, T., Lellouch, E., \& Fornasier, S. 2020, in The Trans-neptunian Solar System, eds. D. Prialnik, M. A. Barucci, \& L. A. Young, 153

Peixinho, N., Thirouin, A., Tegler, S. C., et al. 2020, in The trans-neptunian solar system, eds. D. Prialnik, M. A. Barucci, \& L. A. Young, 307

Poston, M. J., Mahjoub, A., \& Ehlmann, B. L. 2018, ApJ, 856, 124

Rauer, H., Helbert, J., Arpigny, C., et al. 2003, A\&A, 397, 1109

Ridden-Harper, R., Bannister, M. T., \& Kokotanekova, R. 2021, RNASS AAS, 5,161

Roth, N. X., Milam, S. N., Cordiner, M. A., et al. 2021, ApJ, 921, 14

Rousseau, B., Erard, S., Beck, P., et al. 2018, Icarus, 306, 306

Schambeau, C. A., Fernández, Y. R., Samarasinha, N. H., et al. 2021, PSJ, 2, 126

Spencer, J. R., Lebofsky, L. A., \& Sykes, M. V. 1989, Icarus, 78, 337

Stansberry, J. A., van Cleve, J., Reach, W. T., et al. 2004, ApJS, 154, 463

Szabó, Gy. M., Sárneczky, M., \& Kiss, L, L. 2008, ApJ, 677, L121

Szabó, Gy. M., Sárneczky, M., \& Kiss, L. L. 2011, A\&A, 531, A11

Szabó, Gy. M., Kiss, L. L., Pál, A., et al. 2012, ApJ, 761, 7

Wong, I., Mishra, A., \& Brown, M. E. 2019, AJ, 157, 225

Zakharov, V. V., Ivanovski, S. L., Crifo, J.-F., et al. 2018, Icarus, 312, 121 


\section{Appendix A: Absolute flux calibration}
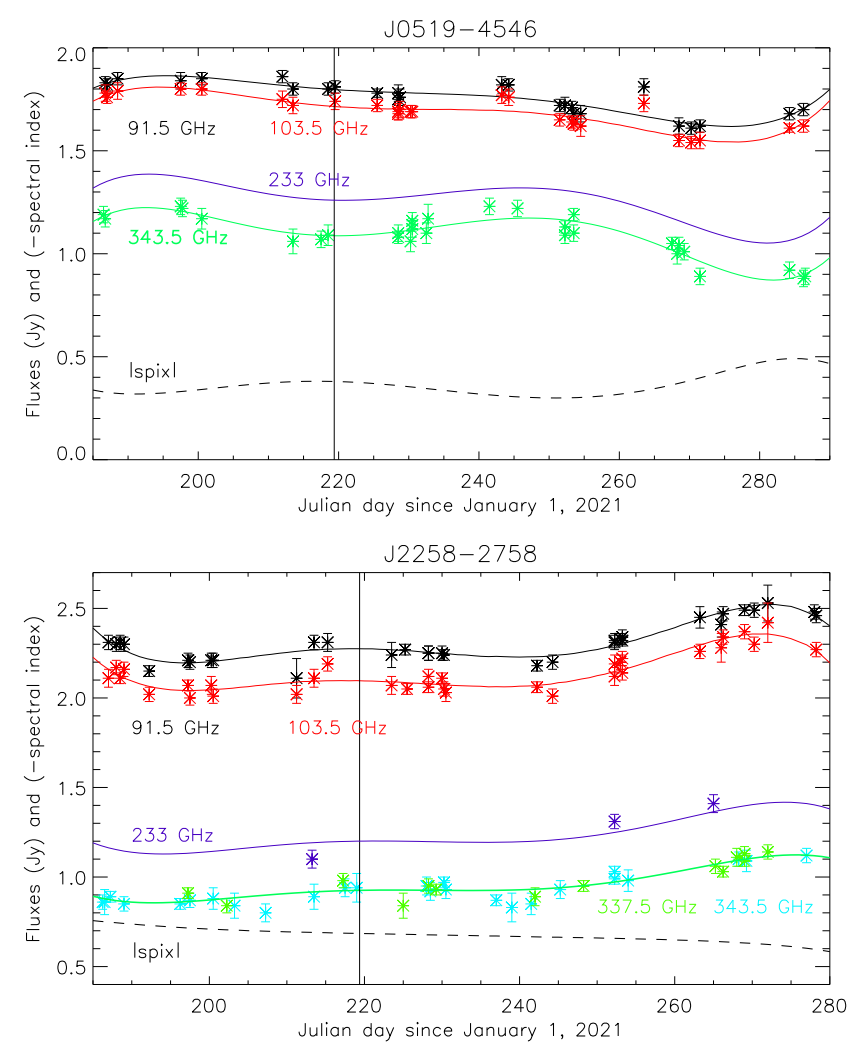

Fig. A.1. Flux measurements of the two flux (bandpass) calibrators over a period of $\sim 100$ days that spans our observation period. These measurements, color-coded by frequency, are taken from the ALMA Calibrator Source Catalogue at $91.5,103.5$, and 337.5 or $343.5 \mathrm{GHz}$. The solid lines are polynomial fits, from which spectral indices and the $233 \mathrm{GHz}$ flux as a function of time are inferred. In the case of J2258-2758, a few direct $233 \mathrm{GHz}$ measurements are also available and plotted. The dashed line, labelled "|spix|," shows the absolute value of the spectral index. The vertical bar corresponds to the date of the $2014 \mathrm{UN}_{271}$ observations.

A good knowledge of the calibrators is critical for the reliability of the flux scale. Our flux (bandpass) calibrators, J2258-2758 and J0519-4546 for the first and second SB, respectively, are variable but routinely monitored from ALMA ${ }^{8}$, mostly at 91.5, 103.5 , and $\sim 340 \mathrm{GHz}(337.5$ or $343.5 \mathrm{GHz})$, and for the second one occasionally at $233 \mathrm{GHz}$. These measurements are displayed in Fig. A.1 over a period of $\sim 100$ days that spans our observing period. We fitted the $91.5,103.5$, and $\sim 340 \mathrm{GHz}$ fluxes with fivedegree polynomials, from which the spectral index over this frequency range and the $233 \mathrm{GHz}$ flux was determined as a function of time. Interpolation on the date of our observation then yielded the desired quasar $233 \mathrm{GHz}$ flux. This approach, illustrated in Fig. A.1, yielded 1.26 Jy for J0519-4546 and 1.20 Jy for J22582758 on August 8, 2021. For the latter object, the value is also nicely consistent with the few available $233 \mathrm{GHz}$ measurements. Moreover, J2258-2758 is also monitored at $\sim 225.5 \mathrm{GHz}$ from the Submillimeter Array (SMA) ${ }^{9}$. Interpolating between 225.5 GHz measurements from July 6, July 9, and August 17, 2021, and applying a spectral index of -0.68 (see Fig. A.1) leads to a $233 \mathrm{GHz}$ flux of $1.24 \mathrm{Jy}$, which compares well with the above

\footnotetext{
8 https://almascience.eso.org/sc/

9 http://sma1.sma.hawaii.edu/callist/callist.html
}

$1.20 \mathrm{Jy}$ value. We finally adopt a $1.22 \mathrm{Jy}$ flux for J2258-2758, with an estimated $2 \%$ uncertainty.

As indicated in the main text, each of the two SBs used the same phase calibrator, J0253-5441. This calibrator is not monitored in terms of flux at ALMA. However, calibrating the visibilities on each flux calibrator yielded two independent measurements of its flux, namely 0.374 and $0.389 \mathrm{Jy}$ for the two SBs (Table 1). We adopt the relative difference, $4 \%$, as a conservative estimate on the absolute calibration uncertainty. This uncertainty is dwarfed by the $\mathrm{S} / \mathrm{N}$-limited error bar on the $2014 \mathrm{UN}_{271}$ flux, equivalent to $9 \%$, but was taken into account in the final error bar.

\section{Appendix B: Dust signal estimation}

We estimated the coma flux density at $233 \mathrm{GHz}$ in the ALMA $0.067^{\prime \prime} \times 0.062^{\prime \prime}$ synthesized beam on the basis of reported optical aperture photometry. In practice, we used the $A f \rho$ value of $\sim 150 \mathrm{~m}$ deduced from the observations of $\mathrm{C} / 2014 \mathrm{UN}_{271}$ on June 29, $2021\left(r_{\mathrm{h}}=20.15\right.$ ua; Dekelver 2021, see also Bernardinelli et al. 2021b). The $A f \rho$ parameter, where $A$ and $f$ are the grain albedo and the filling factor of the instrument field of view with projected radius $\rho$, is independent of $\rho$ if the line of sight column density falls off as $1 / \rho$, as expected for steady and isotropic dust production (A'Hearn et al. 1984). The Af $\rho$ parameter is proportional to the dust production rate, with a coefficient of proportionality that depends on the particle size and velocity distributions. Both the independence on $\rho$ and the increase in $A f \rho$ as $2014 \mathrm{UN}_{271}$ approached the Sun from 28 to 20 au is consistent with $A f \rho$ measuring the actual activity rate of the comet (Bernardinelli et al. 2021b).

Our estimation of the coma flux density at $233 \mathrm{GHz}$ is based on two steps: (1) the determination of the dust production rate, $Q_{\mathrm{d}}$, from the measured $A f \rho$ using the Mie scattering calculations of Fink \& Rubin (2012); and (2) the computation of the dust thermal emission using the model of Bockelée-Morvan et al. (2017), also using Mie theory. Calculations were made for size distributions given by $\mathrm{d} Q_{\mathrm{d}}(a) / \mathrm{da} \propto a^{-\beta}$, where $a$ is the particle radius and $\beta$ is the size index. We considered a minimum size of $0.01 \mu \mathrm{m}$ and maximum sizes, $a_{\max }$, of $10 \mu \mathrm{m}$ and $1 \mathrm{~cm}$.

\section{B.1. Dust production rate}

Fink \& Rubin (2012) provide the $Q_{\mathrm{d}} / A f \rho$ ratio $\left(\mathrm{kg} \mathrm{s}^{-1} / \mathrm{m}\right)$ for particle sizes in the range $0.01 \mu \mathrm{m}-1 \mathrm{~cm}$ and various size index values. Their calculations were made for a refractive index $n=$ $2.00+0.10 i$, a phase angle $\phi=40^{\circ}$, and particle velocities following $v(a)=0.1423 a^{-0.5} \mathrm{~m} / \mathrm{s}, a$ being the particle radius in $\mathrm{m}(v=142.3 \mathrm{~m} / \mathrm{s}$ for $a=1 \mu \mathrm{m})$. We rescaled $Q_{\mathrm{d}} / A f \rho$ to a phase angle of $3^{\circ}$, assuming a phase function ratio $p\left(\phi=3^{\circ}\right) / p\left(\phi=40^{\circ}\right)$ $=2.42$. This value matches the composite phase function of $\mathrm{D}$. Schleicher ${ }^{10}$ and is in the range of the values obtained from Mie calculations (Fink \& Rubin 2012). We also rescaled $Q_{\mathrm{d}} / A f \rho$ to a velocity distribution $v(a)=0.083 a^{-0.5} \mathrm{~m} / \mathrm{s}$. Indeed, based on Crifo \& Rodionov (1997), we derived $v=83 \mathrm{~m} / \mathrm{s}$ for $a=1 \mu \mathrm{m}$ for $2014 \mathrm{UN}_{271}$, using a nucleus radius of $68 \mathrm{~km}$, nucleus and dust densities of $500 \mathrm{~kg} \mathrm{~m}^{-3}$ and $1000 \mathrm{~kg} \mathrm{~m}^{-3}$, respectively, and a CO production rate of $4 \times 10^{28} \mathrm{~mol} \mathrm{~s}^{-1}$ emitted in a cone of $45^{\circ}$ half aperture. The assumed CO production rate $\left(1860 \mathrm{~kg}^{-1}\right)$ is reasonable but somewhat arbitrary. An upper limit of $2 \times 10^{28}$ $\mathrm{mol} \mathrm{s}^{-1}$ was derived from $4.6 \mu \mathrm{m}$ NEOWISE observations at $r_{\mathrm{h}}$ $=20.92$ ua (Farnham et al. 2021). Moreover, extrapolating the

\footnotetext{
${ }^{10}$ https://asteroid. lowell .edu/comet/dustphase.html
} 
Table B.1. C/2014 $\mathrm{UN}_{271}$ dust mass loss rate and flux density in ALMA beam.

\begin{tabular}{|c|c|c|c|c|}
\hline $\begin{array}{l}\text { Size } \\
\text { index }\end{array}$ & $a_{\max }$ & $\begin{array}{l}Q_{\mathrm{d}} / A f \rho^{a} \\
(\mathrm{~kg} / \mathrm{s} / \mathrm{m})\end{array}$ & $\begin{array}{c}Q_{\mathrm{d}}\left(\mathrm{UN}_{271}\right)^{b} \\
(\mathrm{~kg} / \mathrm{s})\end{array}$ & $\begin{array}{c}F(233 \mathrm{GHz})^{c} \\
(\mathrm{mJy})\end{array}$ \\
\hline \multirow[t]{2}{*}{3} & $10 \mu \mathrm{m}$ & 0.291 & 43.6 & $9.910^{-6}$ \\
\hline & $1 \mathrm{~cm}$ & 56.9 & 8544 & $1.510^{-2}$ \\
\hline \multirow[t]{2}{*}{3.5} & $10 \mu \mathrm{m}$ & 0.338 & 50.7 & $1.010^{-5}$ \\
\hline & $1 \mathrm{~cm}$ & 9.16 & 1374 & $2.810^{-3}$ \\
\hline \multirow[t]{2}{*}{4.0} & $10 \mu \mathrm{m}$ & 0.425 & 63.7 & $1.110^{-5}$ \\
\hline & $1 \mathrm{~cm}$ & 0.488 & 73.2 & $8.610^{-5}$ \\
\hline
\end{tabular}

${ }^{a}$ Rescaled from values of Fink \& Rubin (2012) (see text). ${ }^{b}$ For $A f \rho=$ $150 \mathrm{~m}$ (Dekelver 2021). ${ }^{c}$ In a $0.064^{\prime \prime}$ beam width at $r_{h}=20.0$ ua and $\Delta$ $=19.68$ ua.

CO production rate of $\sim 4 \times 10^{27} \mathrm{~mol} \mathrm{~s}^{-1}$ of comet C/1995 O1 (Hale-Bopp) measured at $r_{\mathrm{h}}=14$ au (Biver et al. 2002) (assuming a $r_{\mathrm{h}}^{-2}$ dependence) and correcting for the different nucleus sizes (37 km radius for Hale-Bopp; Szabó et al. 2012) yields an expected $\mathrm{CO}$ production rate at $r_{\mathrm{h}}=20$ au for $\mathrm{C} / 2014 \mathrm{UN}_{271}$ of $\sim 7 \times 10^{27} \mathrm{~mol} \mathrm{~s}^{-1}$. Using the nominal $\mathrm{CO}$ production rate of $4 \times 10^{28} \mathrm{~mol} \mathrm{~s}^{-1}$, the rescaled $Q_{\mathrm{d}} / A f \rho$ ratio and the derived dust production rate $Q_{\mathrm{d}}$ for $\mathrm{C} / 2014 \mathrm{UN}_{271}$ are given in Table B.1 for size indices of 3, 3.5, and 4, given the measured $A f \rho$ of $150 \mathrm{~m}$ (Dekelver 2021).

Although we considered $a_{\max }$ of $10 \mu \mathrm{m}$ and $1 \mathrm{~cm}$ to be conservative, the large size of $\mathrm{C} / 2014 \mathrm{UN}_{271}$ makes the release of large particles unlikely. With the adopted $\mathrm{CO}$ gas production rate of $4 \times 10^{28} \mathrm{~mol} \mathrm{~s}^{-1}$, we estimated a maximum liftable size of $8 \mu \mathrm{m}$ following Zakharov et al. (2018). For a CO production rate of $7 \times 10^{27} \mathrm{~mol} \mathrm{~s}^{-1}$, the maximum liftable size is only 1.3 $\mu \mathrm{m}$. Calculations for $a_{\max }=1.3 \mu \mathrm{m}$ were not performed, but that case would obviously lead to even smaller dust production rates and thermal flux densities than those reported in Table B.1 for $a_{\max }=10 \mu \mathrm{m}$.

\section{B.2. Thermal flux of the dust}

To compute the expected dust emission at millimeter wavelengths, we then used the model described in Bockelée-Morvan et al. (2017), which computes the wavelength-dependent absorption coefficient and the temperature of the dust particles as a function of grain size, using Mie theory combined with an effective medium theory, allowing mixtures of different materials to be handled. We considered a matrix of amorphous carbon with inclusions of amorphous olivine with a Fe:Mg composition of 50:50 and set the carbon/olivine mass ratio to unity. This model has also been used to analyze ALMA/Atacama Compact Array (ACA) observations observations of C/2015 ER61 (Pan-STARRS) and mid-infrared data of comet 29P/Schwassmann-Wachmann (Roth et al. 2021; Schambeau et al. 2021). The dust local density is described as in Fink \& Rubin (2012), following a $1 /\left(r^{2} v(a)\right)$ law, where $r$ is the distance to nucleus. The flux density was computed by summing the blackbody thermal emission of individual particles over the size range and field of view, weighted by the particle size distribution. For consistency with the model of Fink \& Rubin (2012), the dust density was taken equal to $1000 \mathrm{~kg} \mathrm{~m}^{-3}$. The computed flux densities in a Gaussian beam of half-power beam width $(\mathrm{HPBW})=0.064 "$ (corresponding to the ALMA synthesized beam) are given in the last column Table B.1 and

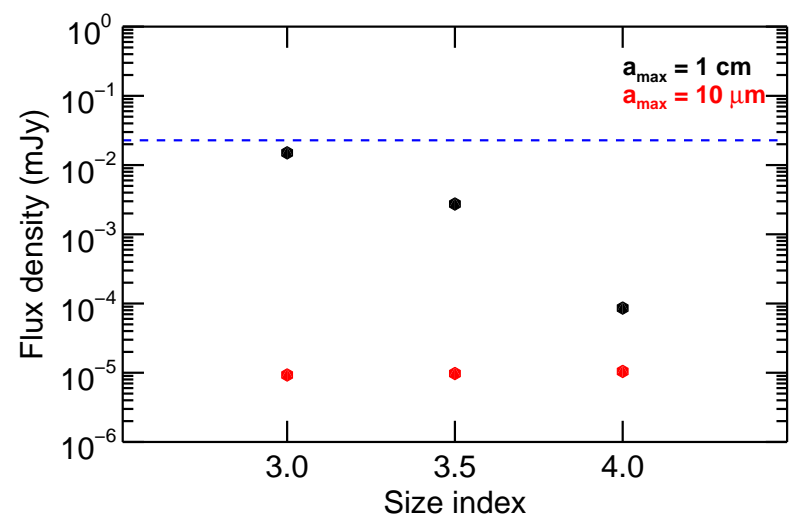

Fig. B.1. Expected flux density at $233 \mathrm{GHz}$ from dust thermal emission, based on the measured Af $\rho$ of $150 \mathrm{~m}$ at 20.15 ua (Dekelver 2021; Bernardinelli et al. 2021b). The beam width is $0.064^{\prime \prime}$. Results are presented for a size index of $3,3.5$, and 4 and for maximum dust sizes of $10 \mu \mathrm{m}$ (red dots) and $1 \mathrm{~cm}$ (black dots). See text for details. The dashed blue line shows the upper limit derived from ALMA observations.

shown in Fig. B.1. We note that the derived flux values are not dependent on the adopted scaling constant in the dust velocity law, since the same velocity law is used for the $Q_{\mathrm{d}} / A f \rho$ and flux calculations.

\section{B.3. Visibility curve for dust thermal emission}

For comparison with the measured visibility curve (Fig. 2), we also calculated the dust signal in terms of the visibility flux at $220 \mathrm{k} \lambda$. In their Appendix A, Bockelée-Morvan et al. (2010) provide formulas describing how the amplitude of the visibility, $V$, varies as a function of UV radius, $\sigma$, for a brightness distribution varying as $1 / \rho$. This includes the formula for the visibility at $\sigma=0 \mathrm{~m}$, corresponding to the signal in the primary beam. The formulas are given for molecular lines but can be applied to the dust coma by adjusting the factors describing the emission mechanism. From Eqs. A.3 and A.4 of Bockelée-Morvan et al. (2010):

$V(\sigma)=K \frac{c}{\sigma v}$

for $\sigma / D_{\text {beam }} \gg 0.2$, where $D_{\text {beam }}$ is the HPBW of the primary beam, and

$V(0)=K \sqrt{\frac{\pi}{4 \ln (2)}} \pi \Phi_{\text {beam }}$,

where $\Phi_{\text {beam }}$ is the HPBW of the primary beam, this time in radians ${ }^{11}$. Here, $K$ is a constant that incorporates factors related to the emission mechanism and the distance of the comet to the observer. Equation B.2 is derived from Eq. A.4 of Bockelée-Morvan et al. (2010), here with the assumption of a Gaussian beam. Equation B.2 can also be used for the signal in the synthesized interferometric beam, $F_{\text {synth }}$, replacing $\Phi_{\text {beam }}$ with the angular size of synthesized beam, $\Phi_{\text {synth }}$. Hence,

$\frac{V(\sigma)}{F_{\text {synth }}}=\frac{c}{\sigma v} \sqrt{\frac{4 \ln (2)}{\pi}} \frac{1}{\pi \Phi_{\text {synth }}}=\frac{\lambda}{\sigma} \sqrt{\frac{4 \ln (2)}{\pi}} \frac{1}{\pi \Phi_{\text {synth }}}$.

From this formula, the visibility flux at $220 \mathrm{k} \lambda$ from the dust coma is 4.40 times the flux in a $0.064^{\prime \prime}$ beam.

${ }^{11}$ For a uniform circular aperture with the same HPBW, the $\sqrt{\frac{\pi}{4 \ln (2)}}=$ 1.064 term would be replaced by 1 . 
Based on Table B.1 and Fig. B.1, it appears that in virtually all cases the expected thermal emission of dust is entirely negligible. Only in one case (size index $\beta=3, a_{\max }=1 \mathrm{~cm}$ ) does the dust contribution amount to $\sim 12 \%$ of the measured signal in the interferometric beam ${ }^{12}$. Another way of seeing it is that this extreme case corresponds to a visibility of $0.015 \times 4.4=0.066 \mathrm{mJy}$ at $220 \mathrm{k} \lambda$, well within the $3 \sigma$ measured upper limit of $0.1 \mathrm{mJy}$. The latter value corresponds to an upper limit of the dust contribution to the synthesized beam flux of $0.1 / 4.4=0.023 \mathrm{mJy}$, which is plotted in Fig. B.1. We note finally that although Farnham et al. (2021) argued that the coma of $2014 \mathrm{UN}_{271}$ consists mostly of submillimeter-sized particles emitted at low velocities, this does not appear consistent with our estimate of the maximum liftable size. Even if this were the case, the contribution of the coma to the thermal flux would be negligible, based on the interpolation between the $a_{\max }=1 \mathrm{~cm}$ and $a_{\max }=10 \mu \mathrm{m}$ cases in Table B. 1 and Fig. B.1. 